\title{
Valeur adaptative de la pigmentation cutanée chez l'Homme
}

\author{
R. RIQUET \\ Laboratoire d'Anthroplogie \\ Université de Bordeaux I \\ 33405 Talence
}

\section{Résumé}

L'importance de la pigmentation mélanique est, a priori, suggérée par :

- la naissance des mélanoblastes près des crêtes neurales.

- leur migration par le système réticulo-endothélial (immuno-compétent),

- les liens entre les précurseurs de la mélanine et les hormones thyroïdiennse et médullosurrénaliennes.

La valeur adaptative de la pigmentation humaine n'est prouvée que pour les fonctions qui suivent :

- protection contre les brûlures, par les U.V. solaires, dont l'intensité croît avec la dépigmentation (gravité chez les albinos),

- régulation de la synthèse cutanée de la vitamine $\mathrm{D}$ (rachitisme des pigmentés dans les climats non lumineux).

La protection mélanique contre les cancers cutanés est probable. Elle est possible, par voie indirecte, contre certaines infections et parasitoses.

Contrairement à une opinion assez répandue, les anthropologues pensent que la couleur de la peau a joué et jouera longtemps un certain rôle dans l'histoire humaine. Certains, comme l'auteur de ces lignes, conscients de l'extrême importance du revêtement cutané chez tous les êtres vivants et de la valeur très générale des pigments organiques, seront beaucoup plus affirmatifs mais à condition d'intégrer la fonction pigmentaire cutanée dans l'ensemble de la physiologie tégumentaire spécifique et, par voie de conséquence, de séparer la mélanine pilaire de la mélanine épidermique. De toute évidence cette dernière intéresse l'homme au premier chef, la seconde concernant essentiellement l'animal. Pour autant, rien n'est très clair ni très simple en la matière. 


\section{Hypothèses orthoadaptatives}

\section{1. - La mélanine protège contre les brûlures solaires}

\section{1. - Les arguments}

Les brûlures solaires sont d'autant plus fréquentes et plus graves que la peau est plus claire. Pratiquement inconnues parmi les populations à peau noire elles apparaissent quasi-constantes chez les blonds surexposés. Le maximum de gravité se trouve chez les albinos.

Chez les Noirs vitiligineux les plaques achromiques sont sensibles à l'insolation.

Le bronzage progressif en intensifiant l'écran mélanique assure une bonne protection puisqu'il permet de dépasser 40 fois le seuil de tolérance de la peau «vierge en première exposition». Mais l'aptitude au bronzage des blonds reste en général très inférieure à celle des bruns. Il y aurait lieu de quantifier ces données médicales, si banales et peu contestables qu'elles soient, moins pour confirmer que pour affirmer (DAvid et al., 1978).

Si l'épiderme du Noir absorbe 70 p. 100 des U.V. et n'en réfléchit que 25 p. 100 alors que le Blanc en réfléchit 60 p. 100 pour n'en absorber que 25 p. 100 on pourrait croire que c'est le Blanc qui est le mieux protégé contrairement à ce qu'on a dit. Mais il faut ajouter une remarque capitale. Le Noir ne laisse passer que 5 p. 100 des U.V. reçus, au-delà de l'épiderme. Autrement dit la masse des U.V. est absorbéc dans l'épiderme. Chez le Blanc, 15 p. 100 des U.V. reçus passent dans le derme et sont par conséquent capables de provoquer des dégats au niveau des couches superficielles du derme si riches en plexus vasculaires et nerveux. Au total la peau blanche réfléchit bien mais filtre mal alors que la peau noire réfléchit mal mais filtre trois fois mieux que la peau blanche. On verra qu'il faut encore faire intervenir des processus plus finement discriminants.

Il y a parallélisme géographique entre l'intensité de la couleur de la peau et celle des ultra-violets. C'est Fleure (1945) qui eut, le premier, l'idée de porter l'attention davantage sur les ultra-violets que sur la totalité des radiations lumineuses comme on le faisait jusqu'alors bien que dès 1925 P.R. PEACOCK ait déjà correctement posé la question. WaLTER (1958) en poursuivant des recherches dans ce sens a trouvé un taux de corrélation de $0,37 \pm 0,044$ entre l'intensité des U.V. et celle de la couleur des téguments humains. Ce taux n'est pas très élevé bien que non négligeable. Sá relative faiblesse s'explique aisément : les races actuelles sont loin d'occuper les territoires d'origine où les processus sélectifs ont fixé leur génome. 11 y a toutefois bonne concordance pour la majeure partie de l'Afrique, de l'Inde méridionale, de la Mélanésie et de l'Australie.

\section{2. - Les mécanismes explicatifs}

On sait que les U.V.A., de longueur d'onde située entre 320 et 400 nanomètres, agissent sur les mélanocytes dès la sixième heure qui suit l'exposition et pendant plusieurs jours. Il y a là, à la fois accélération de la synthèse de la mélanine et du 
transfert des mélanosomes vers les kératinocytes. Les mélanosomes viennent former devant le noyau des kératinocytes une sorte de chapeau protecteur orienté en fonction des photons reçus. Ce chapeau paraît beaucoup plus important chez les Mélanodermes que chez les Leucodermes, moins par une augmentation du nombre des mélanosomes, très incertaine, que par un accroissement de leur volume et que par une meilleure dispersion. En effet chez les Leucodermes les mélanosomes ont tendance à rester groupés dans des formations plus ou moins vacuolaires entravant les regroupements et de surcroît, ne favorisant peut-être pas une aussi bonne conservation (Quevedo et al., 1975).

L'énergie des photons, transformée en énergie chimique, accélère la multiplication des kératinocytes et par là même l'épaississement de la couche cornée dont le rôle protecteur est affirmé par un nombre croissant de chercheurs (CÉSARINI en particulier).

Dans le cas des U.V.B, de 240 à $300 \mathrm{~nm}$ de longueur d'onde, les processus de protection sont débordés. Bien que fortement accélérée, la production de mélanine n'est plus suffisante et surtout l'unité fonctionnelle qui unit le mélanocytes aux kératinocytes voisins est trop perturbée pour que s'effectue un transfert correct du pigment. Des altérations cytoplasmiques et nucléaires se produisent dont les plus graves conduisent à des fractures des chaînes d'A.D.N. nucléaire. Il en découle non seulement des difficultés de restauration cellulaire et de réplication mais aussi des aberrations chromosomiques éventuellement cancérigènes. On sait combien les albinos y sont exposés. A ce sujet, CÉSARINI signale que chez les Ecossais passant des brumes des Iles Britanniques aux cieux tropicaux de l'Australie le taux de cancers cutanés, sur les régions exposées au soleil, se multiplie par 10 ce qui prouve bien l'action actinique. Mais en Australie ces mêmes sujets ont 100 fois plus de cancers cutanés que les Aborigènes ce qui prouve bien le rôle protecteur de la peau noire. Toutefois les cancers cutanés sont trop rares pour peser d'un grand poids sélectif.

Par contre, dans le cas des U.V.B les photons qui ne sont plus absorbés au niveau de l'épiderme vont altérer le derme au point de provoquer une véritable désorganisation tissulaire : congestion vasculaire, œdème, destruction fibrillaire, formation de plans de clivage, etc. Ces brûlures invalidantes, douloureuses et candidates privilégiées aux infections graves ont eu une valeur sélective certaine, en faveur des Noirs intertropicaux, avant que les Blancs ne développent un microclimat vestimentaire approprié. Et encore nous laissons de côté le syndrome encéphalique d'insolation avec congestion des méninges et œdème cérébral qui fut tant redouté des premiers colons blancs.

\section{3. - Conclusions}

La peau noire constitue une bien meilleure adaptation aux agressions actiniques que la peau blanche.

Par épurations sélectives successives, les aptitudes mélanocytaires des races noires ont été fixées dans le génome. Dans les mêmes conditions les mélanosomes des Australiens et des Noirs africains sont de deux à quatre fois plus longs que ceux des Blancs (SzABo et al., 1969). En outre, si on trouve des populations blanches ayant une peau très foncée, par exemple chez les paysans turcs ou iraniens durant l'été, cette peau reste claire dans les parties couvertes et de toutes façons la pigmentation 
disparaît en hiver ou en zone urbaine. C'est un simple phénomène d'acclimatation dont on a parfois abusé pour diminuer la distance génétique entre Noirs et Blancs.

Si l'humanité a pris naissance dans la zone intertropicale, comme semble le confirmer toutes les découvertes paléanthropologiques de ces dernières années, nos ancêtres ne pouvaient avoir qu'une peau foncée. Elle n'était toutefois probablement pas aussi sombre que celle des noirs actuels si Homo habilis, voire Homo erectus, avait conservé une notable couverture pileuse protectrice. N'oublions pas que le fœtus humain a plus de follicules pileux que les anthropoïdes actuels. Même si nous ne croyons pas que l'ontogénie soit toujours le raccourci de la phylogénie, il faut bien en tenir compte.

La mélanine possède certainement d'autres fonctions que celles qui précèdent, soit par elle-même, soit par un rôle photo-sensibilisateur, soit comme précurseur, soit comme antagoniste.

\section{2. - L'écran mélanique règle la production de vitamine $D$}

\section{1. - Les arguments}

Depuis plus d'un demi-siècle les médecins ont remarqué et utilisé l'action du soleil puis des U.V. sur le rachitisme avant même que la vitamine $D$ ait été séparée de la vitamine A (A. Windaus, 1925).

Contrairement à ces faits, du moins en apparence, les médecins coloniaux trouvèrent d'assez nombreux cas de rachitisme chez les enfants africains, principalement en milieu urbain. On ne pouvait plus retenir la seule action de l'insolation, nullement déficitaire dans ce cas mais celle d'autres facteurs dont la qualité de la peau. Le rôle de cette dernière comme lieu de production de provitamine $\mathrm{D}$ avait d’ailleurs été assez vite reconnu (H. BRocKman, 1936).

De nombreuses confirmations d'une corrélation entre le rachitisme et la suuleur de la peau sont alors apparues.

EASTMAN (1956) qui s'est attaché aux stigmates rachitiques de l'adulte, a constaté que les femmes noires des U.S.A., comparativement aux femmes blanches, présentaient 7 fois plus de bassins ovalaires étroits. Il en résulte 2 à 3 fois plus de présentations anormales du fœtus, 4 à 5 fois plus de procidences du cordon. La fréquence des césariennes et de la mortalité à l'accouchement est 1 fois et demie plus élevée. Dans un milieu aussi médicalisé que celui des U.S.A. ces différences, par ailleurs statistiquement significatives par suite du volume de l'échantillon, n’ont pas de portée démographique. Il n'en serait sans doute pas de même en milieu naturel mais l'argument devient difficile à manier puisque le milieu naturel des Noirs américains ne saurait être que l'Afrique avec beaucoup moins de rachitisme et un mode de vie moins artificiel donc favorable à l'accouchement normal qui est de règle sur ce continent. Retenons donc seulement que les Noirs sont plus exposés au rachitisme dans les climats tempérés extra-tropicaux. CÉSARINI rappelle en outre que de nombreux enfants noirs furent dirigés vers la Suède par la Croix-Rouge en 1946. Presque tous ces enfants développèrent du rachitisme. Il y en avait plus de 200. 
A l'inverse MAYNE (1973) s'est attaché aux immigrants européens de médiocre statut économique installés à Melbourne. Près de 75 p. 100 des cas de rachitisme de la ville provenaient de cette classe pauvre en majorité d'origine méditerranéenne et assez fortement pigmentée. L'enquête médico-sociale a montré que les enfants rachitiques passaient le plus clair de leur vie en appartement dans les étages supérieurs des buildings sans que les parents aient le temps et la possibilité matérielle de les promener. Dans les mêmes conditions les enfants d'immigrés d'Europe Septentrionale ne faisaient presque pas de rachitisme. Une peau claire a donc besoin de moins de soleil.

\section{2. - Mécanismes explicatifs}

On sait qu'à la surface de la peau se trouve un film lipidique riche en stérols, d'origine sébacée pour l'essentiel. L'irradiation de ces stérols conduit à une première vitamine $\mathrm{D}$, inactive, qui reçoit le plus souvent le nom de provitamine. Absorbée par les liquides intercellulaires (?) de couches épidermiques elle rejoint les plexus vasculaires du derme supérieur d'où elle est conduite au foie qui la transforme une première fois. Mais c'est seulement après son passage dans le rein qu'une nouvelle transformation conduira à la vitamine $\mathrm{D}$. Nous ne rappelons ces données classiques et bien étayées que pour signaler, d'une part, la complexité des processus et, d'autre part l'importance de la peau dans la première phase.

Dans la mesure où la peau des hommes de race blanche se laisse plus facilement traverser par les U.V. que celle des hommes à peau noire, dont la mélanine absorbe l'énergie des photons, l'irradiation des stérols épidermiques est logiquement beaucoup plus importante chez les premiers. Elle se poursuit jusque dans le derme. Il reste par contre difficile d'apprécier le rôle réflecteur de la peau dans les processus chimiques les plus superficiels et peut-être les plus importants.

Loomis 1967) a exploité, dans un sens anthropologique, le rôle de la peau dans la production de la vitamine D. Pour cet auteur, un europoïde exposé pendant 6 heures à un intense soleil pourrait synthétiser jusqu'à 800000 unités (U.I.) de vitamine D. Or cette dose conduit à des troubles nerveux graves, souvent mortels. C'est pourquoi les peaux blanches auraient été éliminées, par la sélection, de tous les pays tropicaux. Les Mongoloïdes et Amérindiens qui ont colonisé secondairement des pays tropicaux auraient développé un type de protection différent de celui des Noirs par épaississement de la couche cornée.

Cette conception «évolutive», si intéressante qu'elle soit, appelle un certain nombre de mises en garde.

Notons tout d'abord que l'intoxication spontanée par surproduction de vitamine D n'a jamais été observée dans la nature, tout au moins à notre connaissance.

En outre l'insolation excessive commence à produire ses effets désagréables bien avant la sixième heure, contrairement à l'insolation modérée. Le sujet, alerté, trouvera rapidement une protection par quelque artifice et instruit par l'accident de brûlure solaire il saura vite en éviter la répétition. L’Homme n'est pas inerte à ce point.

Le bronzage spontané de nombreux Soudanais ou d'Amérindiens des plateaux du Mexique ou du Colorado semble suffisant pour les protéger contre les effets 
nocifs des U.V. sans qu'il faille invoquer un épaississement de la couche cornée. Le rôle de cette dernière reste d'ailleurs très discuté encore que certains comme CÉSARINI lui attribuent une réelle valeur chez les albinos. Mais c'est un cas assez particulier.

Notons enfin qu'il existe une certaine homéostasie entre les ions $\mathrm{Ca}$ et $\mathbf{P}$ ce qui met l'organisme à l'abri de bien des accidents hypercalciques. Il est vrai qu'il n'y a point de défense qui ne puisse être débordée.

\section{3. - Conclusions}

Il faut modifier les vues de Loomis. Les Blancs n'ont pas été éliminés d'Afrique par une sursynthèse pathologique de vitamine $D$. C'est au contraire la sélection accompagnant l'expansion de nos plus lointains ancêtres vers des régions moins ensoleillées qui peut seule rendre compte d'un éclaircissement progressif de la peau vers les pays où le taux des U.V. reçues ne permettait pas une ossification correcte pour les peaux noires.

La dépigmentation date au moins de 30000 ans, date où fut peuplée l'Amérique, continent où on ne trouve pas de Mélanodermes indigènes. On peut la supposer sensiblement plus ancienne. En effet les Néandertaliens du Würmien bénéficiaient forcément d'une certaine adaptation à l'ambiance préglaciaire. Ce n'est pas faire preuve d'une audace excessive que de les supposer dépigmentés dans les régions froides et nuageuses.

Le balancement entre les inconvénients et les avantages d'une peau claire et d'une peau foncée illustre parfaitement le caractère polymorphe des processus adaptatifs.

\section{Hypothèses alloadaptatives}

\section{1. - La défense anti-infectieuse pourrait utiliser certains facteurs mélanisants}

\section{1. - Mélanine et système réticulo-endothélial}

Comme les mélanoblastes migrent par le biais du système réticulo-endothélial on peut supposer une certaine parenté entre les deux.

Pour certains auteurs (SCHEER, 1964) le système réticulo-endothélial des Noirs serait, en effet, plus actif que celui des Blancs :

- phagocytose plus importante,

- gamma globulines plus élevées.

\section{2. - Mélano-stimuline et cortico-stimuline (A.C.T.H.)}

On note une certaine parenté chimique entre la mélano-stimuline $\alpha$ et l'A.C.T.H. Elles ont en commun une séquence de 13 acides aminés. A noter que la fin de l'M.S.H. (mélano-stimuline) est acétylée. L'A.C.T.H. de synthèse a 23 acides aminés 
(38 pour la naturelle) acquiert par acétylation une action mélanotrope mais au détriment de sa fonction corticotrope. Il se peut qu'il y ait une sorte de balancement antagoniste entre les deux hormones (LEE et al., 1963; MC QUIRE \& LERNER, 1963).

Il y aurait chez les Noirs prévalence de la fonction mélanotrope sur la fonction corticotrope ce qui expliquerait au moins en partie la moins grande activité de la cortico-surrénale chez les Noirs prouvée par le taux des 17 céto-stéroïdes, celui des éosinophile, la rétention potassique, la courbe d'hyperglycémie provoquée, etc. Comme l'action anti-inflammatoire de l'A.C.T.H. et du Cortisol gène l'efficacité de la défense anti-infectieuse le Noir serait avantagé. Il semble effectivement moins sensible aux maladies tropicales et par là même mieux adapté que le Blanc à son milieu d'origine si riche en parasites, bactéries et virus. Pour Leguebe (1977) il y aurait là un facteur d'adaptation plus important que l'écran mélanique.

On ne peut toutefois conclure nettement car la longue tolérance des Noirs à la tuberculose et à l'infection des séreuses, classiquement admise, évoque plutôt la prévalence des anti-inflammatoires (A.C.T.H. et Cortisol). Une seule chose paraît certaine, le comportement du Noir devant les maladies infectieuses n'est pas exactement le même que celui du Blanc dans les mêmes conditions (RIQuET, 1979).

\section{2. - Les processus mélanisants jouent un rôle auprès du système nerveux}

La genèse des mélanoblastes, à la base des crêtes neurales, la charge en mélanine des cellules de l'épithélium pigmentaire de la rétine et de la choroïde aussi bien que la substantia nigra (Locus niger) greffée sur la voie motrice extra-pyramidale évoquent des liens fonctionnels entre neurones et pigments bien que personne ne puisse apporter de précision sérieuse.

De même, personne ne peut expliquer pourquoi, dans la schizophrénie, on a pu trouver de nombreux dépôts de grains de mélanine dans le système réticulo-endothélial, encore lui, et dans divers autres organes. Greiner \& NichOlson (1965) ont supposé qu'il y avait une déficience congénitale en mélanotonine remplacée par une substance hallucinogène voisine. L'hormone mélanotrope privée de son antagoniste produirait une stimulation excessive de mélanosynthèse qui, faute de mieux, remonterait la vieille route traditionnelle des mélanocytes. Cette situation complexe aurait besoin de beaucoup d'éclaircissements.

L'action aggravante de l'hormone mélanotrope sur la maladie de Parkinson est aussi un mystère ce qui n'a pas empêché Kostin et BARBEAU (1973) d'en tirer un profit logique bien qu'indirect. Ils ont en effet associé le facteur hypothalamique inhibiteur de l'hormone somatrope à la 1-Dopa dans cette maladie du système extra-pyramidal. Les deux auteurs précités ont obtenu des résultats spectaculaires chez leurs parkinsoniens.

De ces données disparates bien qu'évocatrices on serait tenté de rapprocher les différences observées entre l'électro-encéphalogramme des Noirs et des Blancs. Selon Gallais et al., le Noir présenterait beaucoup plus de bouffées d'ondes paroxystiques et serait, par contre, fort peu sensible à l'épreuve stroboscopique si incommodante pour le Blanc. Ajoutons que les psychiatres ont remarqué chez les malades mentaux africains des crises délirantes extrêmement violentes et de très courte durée non mémorisées et dont ils ne trouveraient pas l'équivalent chez les Euro- 
péens. Là encore il faudrait des confirmations mais tout ce qui concerne les différences raciales surtout en matière de physiologie cérébrale est désormais tabou. Cette confusion entre physiologie différentielle et morale universelle est tout à fait regrettable.

Malgré l'incontestable intérêt des données qui précèdent il est encore impussible d'en tirer parti en ce qui concerne la sélection des groupes humains.

\section{3. - Les hormones de la fonction mélanique règlent nos rythmes biologiques}

La mélatonine épiphysaire se comporte comme un antagoniste de la M.S.H. (hormone mélanotrope) et plus accessoirement la F.S.H. (hormone de stimulation folliculaire). Or la production de mélatonine est inhibée par la lumière et subit des fluctuations nyctémérales caractéristiques. Toutefois il pourrait s'agir d'un phénomène passif n'intéressant la M.S.H. que de façon tout à fait marginale.

Mais le précurseur de la mélanotonine, la sérotonine, se comporte comme un concurrent de la M.S.H. et sa production est stimulée par la lumière. La sérotonine est en outre un concurrent de la noradrénaline des globules synaptiques des terminaisons du système sympathique lequel est justement intéressé, par le ganglion sympathique cervical supérieur, à la transmission de l'action de la lumière vers la glande pinéale. On entrevoit tout un réseau de relations encore mal définies et d'aspect parfois contradictoire. Par exemple la lumière stimule la production de sérotonine alors que celle-ci joue un rôle de premier plan dans le sommeil.

Là encore il est impossible d'orienter un faisceau d'arguments dans une direction « adaptative». Une voie est cependant ouverte (Quevedo et al., 1975).

\section{Hypothèses à rejeter}

\section{1. - Les téguments foncés protègent contre la chaleur}

Bien que retenue par HamiLton (1973) et beaucoup de classiques antérieurs, dont l'opinion se fondait sur la mauvaise tolérance des Noirs aux climats froids, rien ne prouve le rôle de la couleur de la peau en cette affaire. Au contraire la peau noire absorbe beaucoup mieux les radiations caloriques alors que la peau blanche les réfléchit davantage. Il faut donc, chez le Noir, compenser cet inconvénient par d'autres facteurs (transpiration, circulation dans les extrémités, etc.).

\section{2. - La peau sombre a valeur de camouflage}

Soutenue par Coowles (1950) cette idée, visiblement inspirée de la zoologie, suppose que les premiers Hommes à peau foncée, voire les ancêtres de l'Homme, auraient pris naissance dans la forêt ombrophile. Malheureusement les Noirs les plus noirs sont ceux des savanes qui furent aussi le milieu d'origine des Australopithécinés et de l'Homo habilis. 


\section{Bilan}

On peut retenir que la valeur adaptative de la pigmentation cutanée concerne surtout le bon usage des ultra-violets par une sorte de balancement entre la protection des téguments contre les brûlures solaires et la nécessaire synthèse de la vitamine $\mathrm{D}$.

Les hypothèses alloadaptatives, d'un très grand intérêt, si elles mettent en évidence la variété des impacts de la fonction mélanisante n'autorisent pas encore des vues adaptatives. Nous en sommes toutefois assez proches pour ce qui concerne la défense anti-infectieuse.

Reçu pour publication en mars 1981.

\section{Summary \\ Adaptative value of cutaneous pigmentation in Man}

We can estimate that importance of melanic pigmentation is suggested by :

- the growth of melanoblasts by neural crests,

- their migration by the reticulo-endothelial system (ability to immunization), hormones.

- the links between the precursors of melanin and the thyroidal and medulla surrenal

The adaptability of human pigmentation is only prooved for the following functions :

- protection against U.V. sun burns, of which intensity grows according to the depigmentation (very severe for albino), unsunny areas).

- regulation of cutaneous synthesis of $\mathbf{D}$ vitamin (rachitism of pigmented people in

Melanic protection against cutaneous cancers is probable. It can undirectly act against some infections and parasitic deseases.

\section{Références bibliographiques}

Agache P., 1971. Données nouvelles sur les mélanomes bénins cutanés. Sem. Hop. Paris, $47,19,1219 \cdot 1229$.

Carothers J.C., 1954. Psychologie normale et pathologique de l'Africain. Série O.M.S., Masson, Paris.

CéSARINI J.P., 1968. Contribution à l'étude structurale des tumeurs noires de la peau. Thèse méd., Marseille.

CÉSARINI J.P., 1980. Communications verbales, C.N.R.Z., Jouy-en-Josas.

Coon C.S., 1955. Some problems of human variability and natural selection in climate and culture. Amer. Nat., 89, 257-280.

Cowles R.B., 1950. The black skin and human protective coloration. Journ. Ent. Zool., 42, I-4. 
Cowles R.B., 1951. Some ecological factors bearing on the origin and evolution of pigment in the human skin. Amer. Naturalist, 93, 283-293.

Damon A., 1975. Physiological anthropology. Oxford Univers. Press, Toronto.

David Cl., Maillet M. et Tharanne M., 1978. La pigmentation cutanée. Rev. méd. de Tours, 12, 3, 348-358.

Degos R., 1968. La peau. Encyclopaedia Universalis, 12, 651-658.

Eastman N.J., 1956. Obstetrics, 9" édit., Appleton-Century Crofts, 885-923.

Gallais P., Corriol J., Bert J., 1949. Méd. tropic., 9, 613 et suiv.

Gallais P., Miletto G., Corriol J., Bert J., 1951. Méd. tropic., II, 5 et suiv.

Fleure H.J., 1945. The distribution of types of skin color. Géogr. Rev., 35, 380-395.

Greiner A.C., Nicolson G.A., 1969. Canad. psychr. assoc. journ., 10, 109.

Hamilton W.J., 1973. Life's color code. Mc Graw-Hill, New York.

Kostin A.J., Barbeau A., 1973. Cités par Médecine Mondiale, 3 avril 1973, 45.

Lee T.H., Lerner A.B., BuettNer J.V., 1963. Species differences and structural requirement for melanocyte stimulating activity of melanocyte stimulating hormone. Ann. New York Acad. Sc., 100, 658-668.

Leguebe A., 1975. Etude anthropologique de la pigmentation. Thèse doct. sc., Univers. Libre, Bruxelles.

Leguebe A., 1947. La pigmentation cutanée : caractère adaptatif ou non. Homo, 28, 217-221.

Lerner A.B., 1960. Hormonal control of pigmentation. Ann. Rev. Med., II, 187-194.

LESCHI J., 1952. Pigmentation et fonctionnement surrénalien. Masson, Paris.

LоOмIS W.F., 1967. Skin-pigment regulation of vitamin-D biosynthesis. Science, 157, 501-506.

LoOMIS W.F., 1968. Vitamin-D, sunlight and natural selection. Science, 159, 653.

MC Guire J.S., Lerner A.B., 1963. Effects of tricosapeptide « ACTH» and alpha-melanocyte-stimulating hormone on the skin color of Man. Ann. New York Acad. Sc., 100, 622-630.

Mayne V. Cité par Médecine Mondiale, 3 avril 1973, 14.

NEER R.M., 1975. The evolutionary significance of vitamin-D, skin pigmentation and ultraviolet light. Amer. Journ. Physic. Anthrop., 43, 409-416.

Nicolaus R.A., 1968. Melanins. Herman, Paris.

Quevedo W.C., Fitzpatrick T.B., Pathak M.A., Jimbow K., 1975. Role of the light in human skin color variation. Amer. Journ. Physic. Anthrop., 43, 393-408.

RiQueT R., 1979. Sur la pathologie raciale. Assoc. Internat. Anthrop. Langue Franç, colloque de Calen (1977), 21-42.

Schally A.V., Kastin A., 1969. Commande hormonale activatrice de la sécrétion hypophysiare. Triangle, journ. Sandoz des Sc. méd., 9, 147-153.

SCHREIDER E., 1963. Anthropologie physiologique et variations climatiques. In : Physiologie et psychologie en milieu aride, UNESCO, Paris, 39-76.

Szabo G., Gerald A.B., Pathak M.A., Fitzpatrick T.B., 1969. Racial differences in the fate of melanosomes in human epidermis. Nature, 222, 1081-1082.

Szabo G., Gerald A.B., Pathak M.A., Fitzpatrick T.B., 1972. The ultra structure of racial color in Man. In: Pigmentation: its genesis and biologic control. AppletonCentury Crofts, 23-41.

Szabo G., 1975. The human skin as an adaptative organ. In: Damon A., 39-58.

Tepperman J., 1969. Physiologie endocrine et métabolique. Masson, Paris, 11-31.

Walter H., 1958. Der Zusammenhang von Hautfarben Verteilung und Intensität der ultravioletten Strahlung. Homo, 9, 1-13.

Wasserman H.P., 1974. Ethnic pigmentation. American Elsevier, New York. 